\title{
Type Ia supernovae as extragalactic distance indicators
}

\author{
Brian P. Schmidt \\ Research School of Astronomy and Astrophysics, Australian National University, Canberra, \\ Australia \\ email: brian@mso.anu.edu.au
}

\begin{abstract}
Type Ia supernovae (SNe Ia) are among cosmology's most useful tools for measuring extragalactic distances. Their intrinsic brightness, $M_{V}=-19.2 \mathrm{mag}$, and precision, $\sigma=0.12$ mag, make for a unique combination to precisely probe cosmic expansion from the nearby to the high-redshift Universe. I describe the current state of the art for measuring distances to $\mathrm{SNe}$ Ia - focusing on the current challenges which ultimately limit their precision - as well as prospects for further refinement. I also highlight cosmological applications where they have been especially valuable, and briefly review some future projects which plan to exploit SNe Ia.
\end{abstract}

Keywords. supernovae: general, galaxies: distances and redshifts, cosmological parameters, distance scale 